\title{
Mercurio en cabello de diferentes grupos ocupacionales en una zona de minería aurifera en el Norte de Colombia
}

\author{
Hair mercury levels in different occupational groups in Southern Bolivar \\ (Columbia)
}

Jesús Olivero, Claudia Mendonza, Judith Mestre

Facultad de Ciencias Qufmicas y Farmacéuticas - Universidad de Cartagena - Colombia

\begin{abstract}
Análisis de mercurio en cabello fue realizado a 219 personas que habitan en la principal zona de minería del oro en Colombia, tomando 27 personas de la ciudad de Cartagena, como muestra Control. Para el análisis de los datos, la muestra estudiada se dividió de acuerdo con la ocupación de las personas, encontrándose concentraciones medias ( $\pm \mathrm{DE}$ ) de $5,23 \pm 5,78,2,83 \pm 3,27,2,40 \pm 2,02$ y $1,33 \pm 0,74 \mu \mathrm{g} / \mathrm{g}$ de mercurio en cabelio, para pescadores, mineros, personas dedicadas a otras actividades y grupo control respectivamente. De acuerdo con el análisis de varianza y el test de Newman Keuls, existe diferencia significativa $(p<0,01)$ para las concentraciones de mercurio observadas entre pescadores y los demás grupos. No se presentaron diferencias significativas de mercurio en cabello de acuerdo con el sexo, tampoco se detectó correlación con la edad, no obstante se observó una ligera correlación positiva ( $R=0,15$, $\mathrm{p}<0,01)$ con la frecuencia en el consumo de pescado. Los principales síntomas de intoxicación mercurial encontrados entre la población expuesta fueron cefalea, náuseas, lesiones orales, gusto metálico, pérdida de memoria e irritabilidad.
\end{abstract}

Pelo, quimica. Mercurio, análisis. Mineria.

\section{Introducción}

El estudio se realizó en el Sur de Bolívar, el cual corresponde a una zona geográfica del norte de Colombia, enmarcada en las coordenadas geográficas $7^{\circ} 7^{\prime}$ y $9^{\circ} 18^{\prime}$ latitud norte y $73^{\circ} 43^{\prime}$ y $74^{\circ} 48^{\prime}$ longitud oeste del meridiano de Greenwich. Comprende un área total de $16.503 \mathrm{~km}^{2} \mathrm{y}$ dista $240 \mathrm{~km}$ de la ciudad de Cartagena, su principal actividad económica la constituye la minería del oro, siendo la producción para 1994 de 432.103 O.T, convirtiéndose en la zona de mayor densidad minera de Colombia, con aproximadamente 12.400 minas en explotación'.

La minería aurífera se realiza en forma completamente artesanal empleando mercurio metálico en los procesos de beneficio, involucra a cerca de 33.000 personas que reciben influencia directa e indirecta de esta actividad. Las minas están localizadas cerca a quebradas y ciénagas, estas últimas despensa pesquera de la región y de gran parte de la costa atlántica colombiana ${ }^{6}$.
En virtud de que diariamente los vertimientos de la minería son arrojados a los cuerpos de agua sin ningún tipo de tratamiento, el presente estudio muestra la magnitud de la contaminación por mercurio entre la población del Sur de Bolívar (Colombia) y la incidencia de la misma sobre su salud.

El análisis de mercurio se realizó en cabello dado que éste funciona como dosímetro y la cantidad hallada en él es proporcional a la que ha sido incorporada en el organismo ${ }^{2}$, de otra parte, el cabello constituye una herramienta útil para el monitoreo de mercurio en exposiciones de tipo ocupacional ${ }^{3}$.

\section{Material y Método}

\section{Muestra}

La muestra analizada correspondió a personas que habitan en el Sur de Bolívar, en los municipios de Achí, Río Viejo, San Martín de Loba, Barranco de Loba y Pinillos. Un grupo de 27 personas resi- 
dentes en la ciudad de Cartagena fue tomada como muestra Control. La Tabla 1 señala la distribución frecuencial del total de personas analizadas. Los habitantes del Sur de Bolívar fueron agrupados por ocupación en: Mineros, Pescadores y personas dedicadas a otro tipo de actividad (Otros). La muestra incluyó individuos de diferente sexo y edades que oscilaron entre 2 y 85 años. Los niños y las mujeres fueron ubicados dentro de los grupos a los cuales pertenecían los padres cabeza de familia.

Tabla 1 - Distribución de frecuencias por sexo y edad para los grupos poblacionales estudiados en el Sur de Bolivar y Control.

\begin{tabular}{lrrrrrr}
\hline Grupo & n & \multicolumn{4}{c}{ Frecuencla (\%) } \\
\cline { 3 - 5 } & & \multicolumn{2}{c}{ Sexo } & \multicolumn{3}{c}{ Edad (años) } \\
\cline { 3 - 4 } & & Masc. & Fem. & $\leq 15$ & $15-30$ & $\geq 30$ \\
\hline Mineros & 157 & 57,3 & 42,7 & 22,3 & 39,5 & 38,2 \\
Pescadores & 23 & 39,1 & 60,9 & 17,4 & 17,4 & 65,2 \\
Otros & 39 & 82,0 & 18,0 & 43,6 & 15,4 & 41,0 \\
Control & 27 & 55,5 & 44,5 & 22,2 & 48,2 & 29,6 \\
\hline
\end{tabular}

\section{Recolección de la Muestra}

El muestreo se desarrolló entre abril y junio de 1994. Las muestras de cabello se recolectaron cortando un mechón de pelo lo más cercano posible al cuero cabelludo. El cabello se fijó con cinta adhesiva a papel y se depositó en un sobre hasta el momento del análisis?

Conjuntamente con la recolección del cabello, a cada individuo se le realiz 6 un cuestionario para obtener información sobre edad, sexo, ocupación, hábitos alimenticios y posibles síntomas asociados con contaminación mercurial.

\section{Tratamiento de la Muestra y Análisis}

Una vez en el laboratorio, las muestras de cabello fueron lavadas con detergente y agua. El mercurio se analiz6 por duplicado empleando el equipo de absorción atómica sin llama MAS-50 B COLEMAN, previa digestión con ácido nítrico, sulfúrico y permanganato de potasio, reactivos libres de mercurio. El procedimiento de análisis empleado presentó una detectabilidad mínima $(\mathrm{S} / \mathrm{N}=3)$ en la curva de calibración de $7,14 \times 10^{4} \mathrm{ppm}$ de mercurio.

\section{Análisis Estadístico}

La comparación entre las medias de cada grupo poblacional en la muestra colectada se realizó mediante un análisis simple de varianza" y el Test de Newman Keuls ${ }^{10}$, a un nivel de significancia del $5 \%$. Mediante análisis de regresión lineal" se establecieron correlaciones entre la concentración de mercurio con la edad y el número de días mensuales de consumo de pescado por individuo.

\section{Resultados y Discusión}

Los resultados de los análisis de mercurio en cabello para los cuatro grupos de población estudiados aparecen en la Tabla 2, como se aprecia, las concentraciones medias son mayores en el orden:

\section{Pescadores $>$ Mineros $>$ Otros $>$ Cartagena.}

En el total de las muestras analizadas (Sur de Bolfvar y Cartagena), las concentraciones oscilaron entre 0,11 y $29,2 \mu \mathrm{g} / \mathrm{g}$, con un valor medio de $2,834 \pm 3,368 \mu \mathrm{g} / \mathrm{g}$. Las medias más altas y bajas observadas correspondieron a los grupos Pescadores $(5,32 \pm 5,79 \mu \mathrm{g} / \mathrm{g})$ y Control $(1,33 \pm 0,74 \mu \mathrm{g} / \mathrm{g})$, respectivamente. El coeficiente de variación de la media fue alto para los Mineros y Pescadores, con 115,2 y $108,7 \%$ respectivamente, esta dispersión de la media obedece a la diferencia en tiempos de exposición de los individuos, la variabilidad en los hábitos alimenticios y factores asociados con el metabolismo, de acuerdo con la edad y el sexo.

Según el análisis de varianza, a un nivel de significancia del $5 \%$ existe diferencia significativa entre las medias de mercurio en cabello para los diferentes grupos de población examinados. Para corroborar si la diferencia entre las muestras de los distintos grupos son debidas al azar o si provienen de poblaciones diferentes, se realizó el Test de Newman Keuls, encontrándose que a $\mathrm{p}>0,05$, al comparar los grupos Otros-Control y Mineros-Otros, no existen diferencias significativas entre sus concentraciones medias. No obstante, si se presentaron entre las medias para Mineros-Control $(p<0,01)$ y entre Pescadores y los demás grupos ( $\mathrm{p}<0,01$ ).

Con el objeto de establecer la incidencia estadística de algunos valores analíticos altos, concentraciones superiores a $10 \mu \mathrm{g} / \mathrm{g}$ fueron separadas del análisis estadístico así: (Pescadores: 3 datos $(11,7$,

Tabla 2 - Resultados de análisis de mercurio en cabello en habitantes del Sur de Bolivar y grupo Control.

\begin{tabular}{|c|c|c|c|c|c|}
\hline \multirow{3}{*}{ Grupo } & \multicolumn{5}{|c|}{ Estadistlcos } \\
\hline & \multirow[b]{2}{*}{$\mathbf{n}$} & Promedio & $\begin{array}{c}\text { Desviación } \\
\text { estándar }\end{array}$ & $\begin{array}{c}\text { Valor } \\
\text { máximo }\end{array}$ & $\begin{array}{l}\text { Valor } \\
\text { mínimo }\end{array}$ \\
\hline & & \multicolumn{4}{|c|}{ ( $\mu g$ de mercurio/g) } \\
\hline Mineros & 157 & 2,83 & 3,27 & 28,30 & 0,11 \\
\hline Pescadores & 23 & 5,32 & 5,79 & 29,20 & 0,40 \\
\hline Otros & 39 & 2,40 & 2,02 & 10,30 & 0,31 \\
\hline Sur de Bolivar & $n=219$ & \multicolumn{2}{|c|}{ Prom. $=3,02$, } & \multicolumn{2}{|c|}{ Desv, Est. $=3,52$} \\
\hline Control & 27 & 1,33 & 0,74 & 3,00 & 0,30 \\
\hline Total & $n=246$ & Prom & $=2,83$ & Desv, Es & st. $=3.37$ \\
\hline
\end{tabular}


$28,3$ y $18,46 \mu \mathrm{g} / \mathrm{g})$, Otros: 1 dato $(10,3 \mu \mathrm{g} / \mathrm{g})$, Mineros: 2 datos $(12,15$ y $29,2 \mu \mathrm{g} / \mathrm{g}$ ). Aunque la disminución de las medias fue ostensible, el Test de Newman Keuls arrojó los mismos resultados obteniơos cuando el análisis se realizó al involucrar el total de los datos, incrementándose de hecho, el valor de q, dada la disminución en la desviación estándar.

Algunos trabajos similares al nuestro, reportan concentraciones medias de mercurio inferiores a las obtenidas por nosotros. Echegaray y Gomes ${ }^{2}$, en un estudio realizado en la costa y sierra del Perú sobre 278 personas, hallaron concentraciones de mercurio en cabello en el rango de 0,025 a 2,44 $\mu \mathrm{g} / \mathrm{g}$. Santos et al. ${ }^{\mathrm{B}}$, en un monitoreo desarrollado sobre 217 niños residentes a orillas de los principales ríos del municipio de Cubatão en el Sudeste de Brasil, encontraron una concentración media de $0,82 \pm 0,48 \mu \mathrm{g} / \mathrm{g}$ en muestras de cabello. Oskarsson et al. ${ }^{5}$, quienes trabajaron con cabello de mujeres suecas que vivían en un área contaminada con emisiones de mercurio, obtuvieron una media de $0,27 \mu \mathrm{g} / \mathrm{g}$ con rango de $0,07-0,96 \mu \mathrm{g} / \mathrm{g}$.

No hubo diferencias significativas $(p>0,05)$ entre la concentración de mercurio en cabello y el sexo. Tampoco se observó correlación significativa entre la concentración de mercurio en cabello y la edad.

La Figura muestra la relación entre el número de días que los individuos consumen pescado durante un mes y la concentración de mercurio en cabello. Como se observa, con el incremento en el consumo de pescado se aumenta la concentración de mercurio en cabello, presentándose un coeficiente de correlación pequeño pero positivo $(R=0,15, \mathrm{p}<0,01)$. $\mathrm{La}$ mayor frecuencia mensual de consumo de pescado, encontrada para el grupo de los Pescadores, coincide con la concentración media más alta de mercurio en cabello. Santos et al. ${ }^{8}$ no encontraron diferencias significativas entre los valores medios de mercurio en cabello de niños consumidores y no consumidores de pescados de los ríos de Cubatão. Por su parte, Svensson et al. ${ }^{9}$, aunque trabajo con mercurio en sangre, encontró una correlación estadísticamente signi-

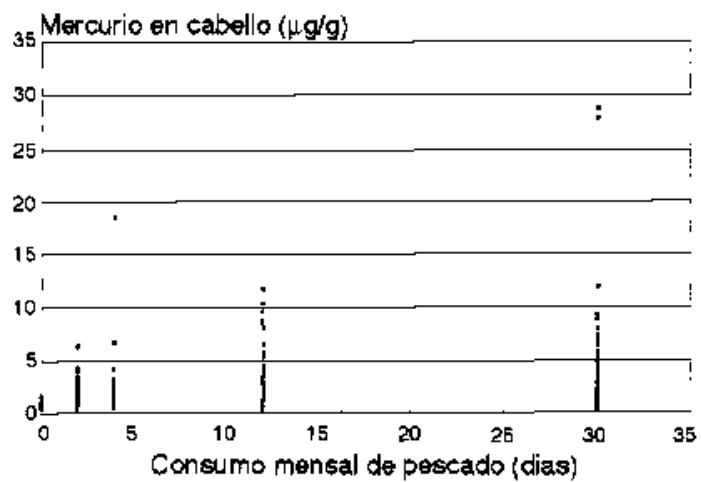

Figura. Relación entre los días mensuales de consumo de pescado y la concentración de mercurio en cabello para habitantes del Sur de Bolivar (Colombia). ficativa entre el consumo de pescado y la concentración de mercurio en una muestra de 395 personas con diversos hábitos de consumo de peces.

En la Tabla 3 se presenta la frecuencia de los principales síntomas asociados con contaminación mercurial encontrados en los diferentes grupos poblacionales del Sur de Bolívar, comparados con el grupo control. Entre los mineros y pescadores del Sur de Bolívar, los síntomas más frecuentes fueron cefalea, pérdida de la memoria, gusto metálico, irritabilidad, náuseas y temblores en las manos, entre otros, resultados parecidos a los reportados por Zavaris y Raah ${ }^{12}$, en un estudio realizado sobre 71 trabajadores expuestos a mercurio metálico en una industria de lámparas eléctricas.

Tabla 3 - Síntomas asociados con intoxicación mercurial. Frecuencia de aparición entre la población del Sur de Bolivar y grupo Control.

\begin{tabular}{|c|c|c|c|c|}
\hline \multirow{2}{*}{ Sintomas } & \multicolumn{4}{|c|}{$\begin{array}{c}\text { Grupos de población } \\
\text { (Frecuencia en Porcentaje) }\end{array}$} \\
\hline & Mineros & Pescadores & Otros & Control \\
\hline $\begin{array}{l}\text { Cefalea } \\
\text { Pérdlda de }\end{array}$ & 52.2 & 43,5 & 46,2 & 18,5 \\
\hline $\begin{array}{l}\text { memoria } \\
\text { Gusto }\end{array}$ & 49,0 & 43,5 & 43,6 & 22,2 \\
\hline metálico & 42,0 & 43,5 & 20,5 & 7,4 \\
\hline $\begin{array}{l}\text { Irritabilidad } \\
\text { Temblores en }\end{array}$ & 42,0 & 30,4 & 17,9 & 3,7 \\
\hline las manos & 35,0 & 30,4 & 20,5 & 0,0 \\
\hline Náuseas & 39,5 & 26,1 & 20,5 & 0,0 \\
\hline $\begin{array}{l}\text { Peladuras } \\
\text { bucales }\end{array}$ & 16,6 & 26,1 & 30,8 & 7,4 \\
\hline $\begin{array}{l}\text { Sangrado } \\
\text { de encias }\end{array}$ & 31,2 & 4,3 & 20,5 & 11,1 \\
\hline
\end{tabular}

El hecho de que la concentración media observada para el grupo control sea cercana a la del grupo otros que habita a gran distancia del primero, además de ser dos veces superior a las reportadas para el grupo control de otro estudio ${ }^{4}$, evidencia la difusión de la contaminación hacia zonas urbanas relativamente alejadas. Esto probablemente obedece a que gran parte del pescado que se consume en Cartagena y otras ciudades de la Costa Atlántica Colombiana proviene de los cuerpos de agua del Sur de Bolívar.

Aunque actualmente las autoridades sanitarias y ambientales están tomando conciencia de la gravedad del problema, es preciso adoptar medidas correctivas inmediatas, especialmente en salud y educación, comenzando por mejorar el nivel de vida de los habitantes del Sur de Bolívar.

\section{Agradecimientos}

A los habitantes del Sur de Bolívar, por la colaboración, y a la Universidad de Cartagena, por el apoyo financiero. 


\section{Referencias Bibliográficas}

1. CENTRO DE ESTLDIOS DE DESARROLLO REGIONAL. Universidad de Cartagena. Plan de Desarrollo Minero del Sur de Bolivar. Cartagena, 1994.

2. ECHEGARAY, $R$, \& GOMEZ, M. Determinación de mercurio en cabello como expresión de la exposición a mercurio. Bol. Lima, 6(31):92, 1984.

3. HAC, E \& KRECHNIAK, J. Mercury concentrations in hair exposed in vitro to mercury vapor. Biol. Trace Elem. Res., 39 (2/3):109, 1993.

4. KOIZLMI, A. et al. Mercury, not sulphur dioxide, poisoning as cause of smelter disease in industrial plants producing sulphuric acid. Lancet, 343: 1411, 1994.

5. OSKARSSON, A. et al. Mercury levels in the hair of pregnant women in a polluted area in Sweden. Sci. Total Environ., 151: 29, 1994.

6. PLAN NACIONAL DE REHABILITACIÓN. Propuesta del Programa de Inversión Social para los nueve municipios del Sur de Bolivar? Secretaría de Planeación Departamental, 1987.
7. PODLESKY, E. \& ORTIZ, Y. Determinación de trazas de metales en muestras biológicas $y$ ambientales: manual de procedimientos. Santa Fe de Bogotá, Sanidad del Ambiente Instituto Nacional de Salud, 1992.

8. SANTOS, E. et al. Teores de chumbo e mercúrio em cabelo de crianças residentes em Cubatão na regiào sudeste do Brasil. Rev. Saúde Publica, 27:81, 1993.

9. SVENSSON, B., et al. Fish as source of exposure to mercury and selenium. Sci. Total Environ, 126:61, 1992

10. TALLARIDA, R. \& MURRAY, R. Manual of pharmacological calculations with computer programs. 2nd ed. New York, Springer-Verlag. 1986.

11. WALPOLE, R. \& MYERS, R. Probabilidad $y$ estadistica. $4^{a}$ ed. México, Ed. Mc.Graw Hill, 1991.

12. ZAVARIZ, C. \& RAAB, M. Avaliação clíniconeuro-psicológica de trabalhadores expostos a mercúrio metálico em indústria de lâmpadas elétricas. Rev. Saüde Püblica, 26:356, 1992.

\begin{abstract}
Hair mercury analysis was carried out on a sample of 219 peopie living in the main gold mining zone of Colombia, 27 inhabitante of Cartagena City being taken as control sample. For data analysis the sample was divided by occupation and the corresponding the hair mercury concentrations (mean $\pm S D$ ) were found to be $5.23 \pm 5.78,2.83 \pm 3.27,2.4 \pm$ 2.02 and $1.33 \pm 0.74 \mathrm{\mu g} / \mathrm{g}$ for fishermen, miners, people of various other activities and the control sample, respectively. According to variance analysis and the Newman Keuls test, there were significant differences $(\rho<0.01)$ between the mercury concentrations for fishermen and those for the other groups. No significant differences were found for hair mercury and sex, non was any correlation with age detected; however, a low positive correlation $(R=0.15, p<0.01)$ with the frequency of the consumption of fish was noted. The main symptons of mercury poinsoning observed in the persons exposed were headache, oral lesions, metalic taste, loss of memory, and irritability.
\end{abstract}

Hair, chemistry. Mercury, analysis. Mining. 